\title{
A bridge method for simultaneous measurements of coupling coefficient and loaded $Q$ of a single-ended cavity
}

\author{
Kenzo Watanabe and Iwao Takao \\ Research Institute of Electronics, Shizuoka University, Hamamatsu, 430, Japan \\ (Received 30 March 1973; and in final form, 26 July 1973)

\begin{abstract}
A bridge method which allows the simultaneous measurements of the coupling coefficient and the loaded $Q$ of a single-ended cavity is described. The measuring system consists of a "magic $T$ " bridge and incident power monitor circuits. Two collinear arms of the magic $T$ are terminated by the cavity under measurement and the built-in short at equal distances. Measurements are made by comparing the reflected power from the bridge with the incident power on the cavity.
\end{abstract}

\section{INTRODUCTION}

The reflectometer method is often used to measure the $Q$ factor of a microwave single-ended cavity. ${ }^{1-3}$ However, the method provides information only about the magnitude of the reflection coefficient; an additional phase measurement is required to collect information of the cavity coupling condition. Three methods have been reported for the coupling coefficient measurement, ${ }^{4-6}$ besides the VSWR method. In either method, the phase measurement becomes difficult, consequently inaccurate, when the coupling coefficient is close to unity.

In this paper, a bridge method is proposed that allows simultaneous measurements of the coupling coefficient and the loaded $Q$ of a single-ended cavity. This method is an extension of the reflectometer method to permit measurements of all cavity parameters without any circuit rearrangement. The measuring procedure is described in detail. The measuring error is also estimated. Although a cavity without coupling loss is treated in the text, the method is applicable to a cavity with coupling loss as described in the Appendix.

\section{MEASURING SYSTEM}

The complete circuit arrangement of the measuring system is shown in Fig. 1. The system consists of two main parts: the microwave bridge circuit employing a "magic T" whose output is detected by detector 1 and the incident power monitor consisting of the calibrated attenuator and detector 2. The two detectors used in the system are a matched pair and are assumed to have square-law response, i.e., their detected outputs are proportional to the incident power.

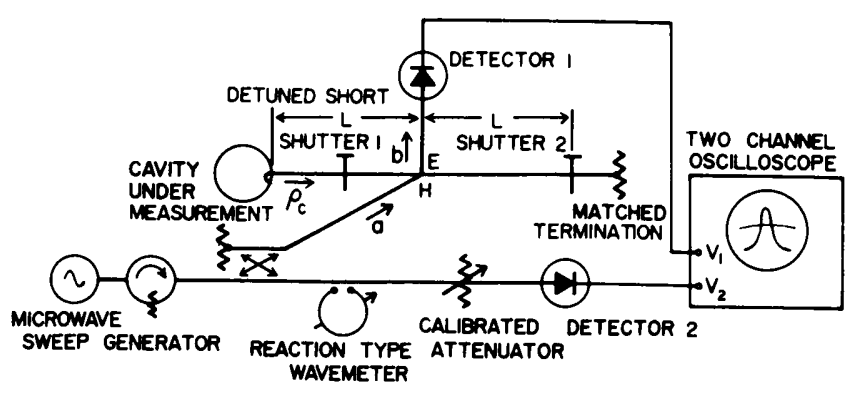

FIG. 1. Circuit arrangement for $Q$ measurement of a single-ended cavity.
The cavity under measurement is connected to one of the collinear arms of the magic $T$. To calibrate the incident power level on the cavity, shutter 1 is placed in front of it. Shutter 2 is connected to the opposite collinear arm at an equal distance from the junction of two collinear arms to the detuned short position of the cavity, which is denoted by $L$ in Fig. 1 .

The signal frequency of the microwave sweep generator is swept through the resonant frequency $f_{0}$ of the cavity. The amplitude $a$ of the incident wave on the magic $\mathrm{T}$ can be written as

$$
a=A \cos 2 \pi\left(f_{0} \pm \Delta f\right) t .
$$

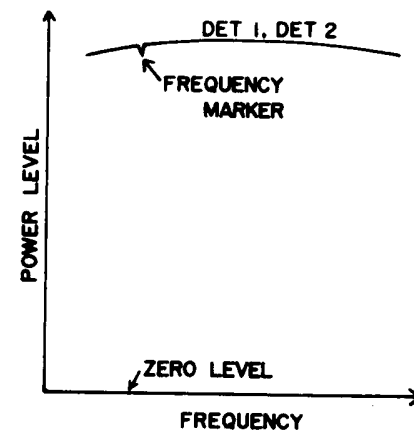

(a)

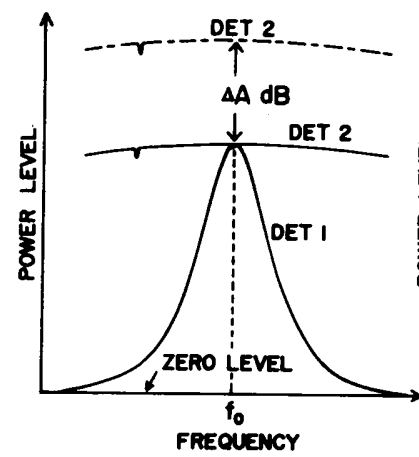

(c)

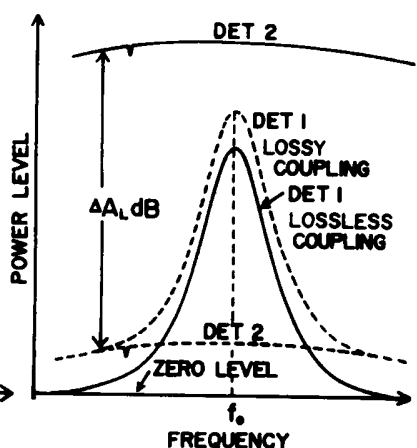

(b)

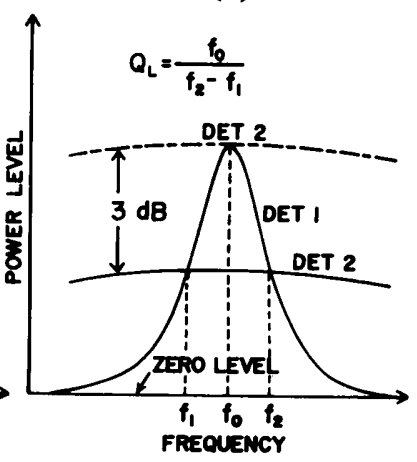

(d)
Fig. 2. Oscilloscope displays of the output levels of detectors 1 and 2 during $Q$ measurement; (a) through (d) correspond to steps (a) through (d) of the measurement procedure.

(a)-During step (a), detector (DET) 2 trace is adjusted to coincide with that of DET 1, which corresponds to the incident power level on the cavity.

(b)-During step (b), DET 1 trace shows the reflected power level. Solid curve-cavity with no coupling loss. Dotted curve-cavity with coupling loss. The coupling loss is measured by adjusting the calibrated attenuator by $\Delta A_{L} \mathrm{~dB}$ until DET 2 trace coincides with DET 1 trace at frequencies removed from $f_{0}$.

(c)-During step (c), DET 2 trace is shifted by adjusting the calibrated attenuator by $\triangle A \mathrm{~dB}$ until it coincides with DET 1 trace at $f_{0}$. (d)-During step (d), DET 2 trace is shifted down by adjusting the calibrated attenuator by $3 \mathrm{~dB}$. The intersections give $f_{1}$ and $f_{2}$. 
FIG. 3. A plot of Eq. (7); coupling coefficient $\beta$ vs attenuator dial reading difference in $\mathrm{dB}$.

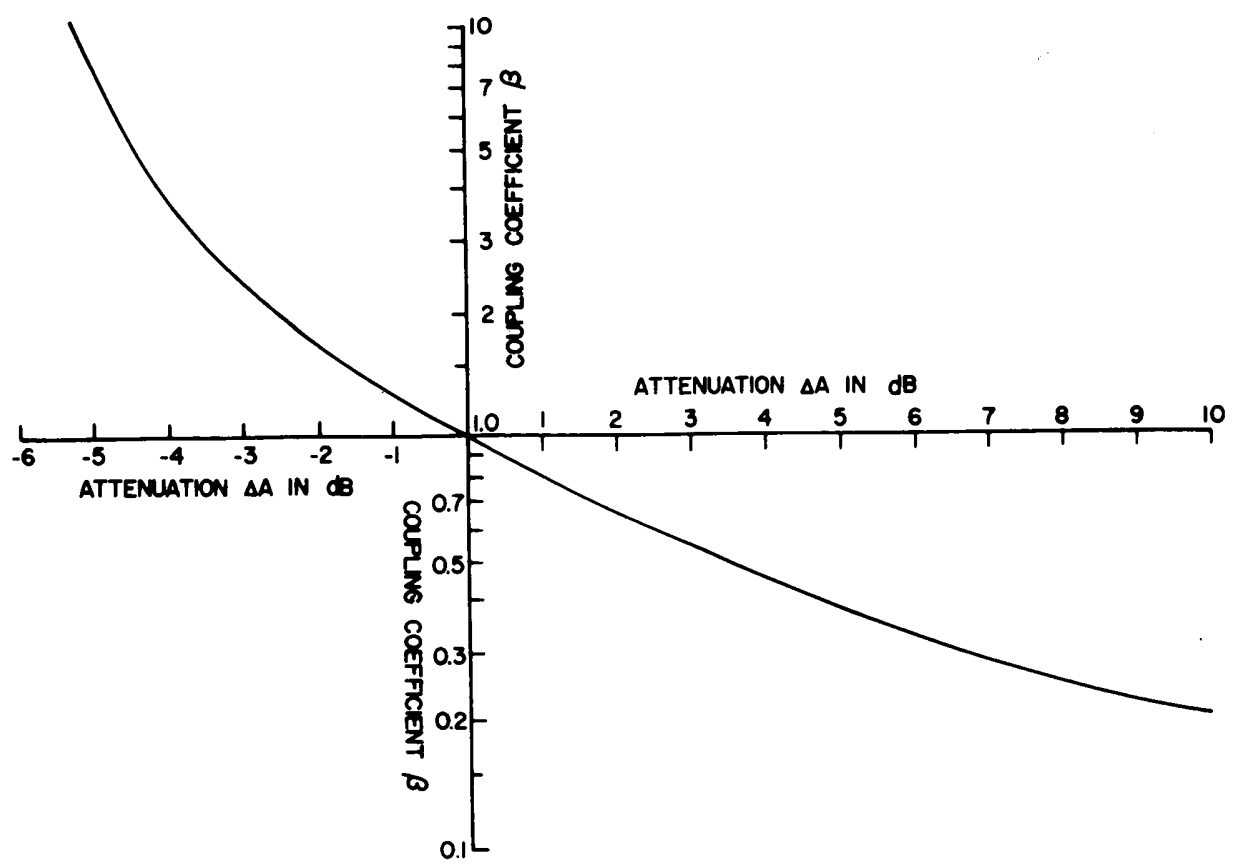

When shutter 1 is closed and shutter 2 is open, the reflected power $\left|\mathbf{b}_{s}\right|^{2}$ to detector 1 is given by

$$
\left|\mathbf{b}_{s}\right|^{2}=A^{2} / 4 \text {. }
$$

When shutter 1 is open and shutter 2 is closed, the reflected power $\left|\mathbf{b}_{c}\right|^{2}$, assuming lossless coupling, is given by

$$
\left|\mathbf{b}_{c}\right|^{2}=\frac{A^{2}}{4}\left|\rho_{c}+1\right|^{2}=\frac{A^{2}}{4} \cdot \frac{[2 \beta /(\beta+1)]^{2}}{1+\left(2 Q_{L} \Delta f / f_{0}\right)^{2}}
$$

where $\rho_{c}$ is the reflection coefficient of the cavity, and is given by

$$
\rho_{c}=\frac{(\beta-1) /(\beta+1)-j 2 Q_{L} \Delta f / f_{0}}{1+j 2 Q_{L} \Delta f / f_{0}}
$$

where $\beta$ and $Q_{L}$ are the coupling coefficient and the loaded $Q$ of the cavity, respectively.

From Eqs. (2) and (3), the following relations are obtained:

$$
\begin{gathered}
\left|\mathbf{b}_{c}\right|^{2} \Delta f=0 /\left|\mathbf{b}_{s}\right|^{2}=[2 \beta /(\beta+1)]^{2}, \\
Q_{L}=f_{0} / 2 \Delta f_{1 / 2},
\end{gathered}
$$

where $\left|\mathbf{b}_{c}\right|^{2} \Delta f=0$ is the reflected power at resonance, and $2 \Delta f_{1 / 2}$ is the half-power bandwidth of $\left|\mathbf{b}_{c}\right|^{2}$.

The power ratio given by Eq. (5) and the half-power bandwidth are measured, respectively, by the calibrated attenuator and the reaction-type wave meter inserted in the incident power monitor circuit.

\section{PROCEDURE OF MEASUREMENT}

Measurements are made in four steps:

Step (a). Close shutter 1 and open shutter 2. The output level of detector 1 shows the reflected power level of $A^{2} / 4$, which is given by Eq. (2). Adjust the incident power on detector 2 with the aid of the calibrated attenuator so that the output level of detector 2 coincides with that of detector 1, as shown in Fig. 2 (a). Record the reading of the calibrated attenuator, say, $A_{1} \mathrm{~dB}$.

Step (b). Open shutter 1 and close shutter 2. The output trace of detector 1 shows the reflected power level, which is symmetrical about the resonant frequency as given by Eq. (3). When the cavity coupling network is lossless, the detected level at frequencies removed from resonance coincides with the zero level, as shown by the solid curve in Fig. 2 (b).

Step (c). Adjust the calibrated attenuator so that the output level of detector 2 coincides with that of detector 1 at resonance, as shown in Fig. 2 (c). Record the new setting of the calibrated attenuator, say, $A_{2} \mathrm{~dB}$. The difference in attenuator readings, $\Delta A=A_{2}-A_{1} \mathrm{~dB}$, is related to the coupling coefficient through Eq. (5) or, in logarithmic form,

$$
\Delta A=20 \log (2 \beta / 1+\beta),
$$

which is plotted in Fig. 3 for convenience.

Step (d). Reduce the incident power on detector 2 by $3 \mathrm{~dB}$ by means of the attenuator from the setting in step (c). The two traces of detector 1 and 2 intersect at

TABLE I. Measured results for parameters of various cavities.

\begin{tabular}{cccccccc}
\hline \hline Method & & \multicolumn{3}{c}{ Proposed bridge method } & \multicolumn{3}{c}{ VSWR method } \\
Cavity & $\Delta A(\mathrm{~dB})$ & $f_{2}-f_{1}(\mathrm{MHz})$ & $f_{0}(\mathrm{MHz})$ & $\beta$ & $Q_{L}$ & $Q_{L}$ \\
\hline $\mathrm{TE}_{102}$ rectangular & 3.8 & 3.2 & 9.428 & 0.47 & 2.920 & 0.44 \\
Reentrant & -1.7 & 8.5 & 9.518 & 1.55 & 1.120 & 1.55 \\
$\mathrm{TE}_{011}$ cylindrical & -4.3 & 4.9 & 9.392 & 4.40 & 1.920 & 4.60 & 1.080 \\
\hline
\end{tabular}


two frequencies $f_{1}$ and $f_{2}$ as illustrated in Fig. 2 (d). The loaded $Q$ is calculated from

$$
Q_{L}=f_{0} /\left(f_{2}-f_{1}\right) .
$$

The parameters of three cavities were measured by the method described above, and the results are tabulated in Table I along with those measured by VSWR method for comparison. Coupling loss was negligibly small in these measurements. Discrepancies between both results are within $\pm 7 \%$.

\section{ACCURACY}

There are three main sources of error in the present $Q$ measuring method. The first is the calibration of the calibrated attenuator and deviation from square-law response of the detectors. The errors arising from them are equivalent to those in the readings of the attenuator dial. Fractional errors in $\beta$ and $Q_{L}$ resulting from the attenuator dial reading error, $\Delta A_{r} \mathrm{~dB}$, are given by, respectively,

$$
\begin{aligned}
\Delta \beta / \beta & =-0.115(\beta+1) \Delta A_{r}, \\
\Delta Q_{L} / Q_{L} & =-0.23 \Delta A_{r} .
\end{aligned}
$$

The second is the improper positioning of shutter 2 . When the discrepancy between the position of shutter 2 and the detuned short position of the cavity is $\Delta L$, fractional errors in $\beta$ and $Q_{L}$ are given approximately by

$$
\begin{gathered}
\frac{\Delta \beta}{\beta}=-\frac{(\beta+1)^{3}}{8 \beta^{2}}\left(\frac{4 \pi \Delta L}{\lambda_{g}}\right)^{2}, \\
\frac{\Delta Q_{L}}{Q_{L}}=\frac{1}{2}\left(\frac{1+\beta}{\beta} \cdot \frac{4 \pi \Delta L}{\lambda_{g}}\right)^{2},
\end{gathered}
$$

where $\lambda_{\theta}$ is the guide wavelength at the resonant frequency.

The third is unequal power split between the two collinear arms of the magic $\mathrm{T}$. When the partition ratio of the wave amplitude between the two collinear arms is $(1-\delta) /(1+\delta)$; fractional errors in $\beta$ and $Q_{L}$ are given approximately by

$$
\begin{gathered}
\frac{\Delta \beta}{\beta}=\frac{(\beta+1)^{2}}{\beta} \cdot \frac{\delta}{16} \\
\frac{\Delta Q_{L}}{Q_{L}}=\left(\frac{\beta+1}{2 \beta}\right)^{2} \cdot \frac{\delta^{2}}{2}
\end{gathered}
$$

If $\Delta A_{r}=0.1 \mathrm{~dB}, \beta=1, \lambda_{\theta}=45 \mathrm{~mm}, \Delta L=0.5 \mathrm{~mm}$, and $\delta=0.05$, the total measurement error in $\beta$ is $3.3 \%$ and that in $Q_{L}$ is $4.2 \%$.

\section{ACKNOWLEDGMENT}

The authors are much indebted to Mr. Ashiki for his help in the experiment. They also thank Dr. S. Mizushina for suggestions on the manuscript.

\section{APPENDIX: MEASUREMENT METHOD AND CALCULATION OF PARAMETERS OF CAVITY WITH COUPLING LOSS}

The reflection coefficient of the cavity with coupling loss can be written as

$$
\rho_{c}=\frac{\gamma_{s}-1+\left(\beta / 1+j 2 Q_{0} \Delta f / f_{0}\right)}{\gamma_{s}+1+\left(\beta / 1+j 2 Q_{0} \Delta f / f_{0}\right)},
$$

where $\gamma_{s}$ is the normalized resistance representing the coupling loss and $Q_{0}$ is the unloaded $Q$ of the cavity. ${ }^{2}$

Introducing the reflection coefficient $\rho_{0}$ at resonance and $p_{1}$ at frequencies removed from resonance, Eq. (A1) is rewritten as follows:

where

$$
\rho_{c}=\frac{\rho_{0}+j 2 \rho_{1} Q_{L} \Delta f / f_{0}}{1+j 2 Q_{L} \Delta f / f_{0}}
$$

$$
\begin{aligned}
\rho_{1} & =\left[\rho_{c}\right]_{\Delta f \rightarrow \infty}=\frac{\gamma_{s}-1}{\gamma_{s}+1}, \\
\rho_{0} & =\left[\rho_{c}\right]_{\Delta f=0}=\frac{\beta+\gamma_{s}-1}{\beta+\gamma_{s}+1}, \\
Q_{L} & =\frac{\gamma_{s}+1}{\beta+\gamma_{s}+1} Q_{0} .
\end{aligned}
$$

Substituting Eq. (A2) into Eq. (3), we obtain

$$
\left|\mathbf{b}_{c}\right|^{2}=\frac{A^{2}}{4} \cdot \frac{\left(1+\rho_{0}\right)^{2}+\left[2 Q_{L}\left(1+\rho_{1}\right) \Delta f / f_{0}\right]^{2}}{1+\left(2 Q_{L} \Delta f / f_{0}\right)^{2}} .
$$

In step (b) of the measuring procedure, the incident power on detector 2 is reduced by means of the calibrated attenuator so that the detected level of detector 2 coincides with that of detector 1 at frequencies removed from resonance as shown by the dotted curve in Fig. 2 (b). The difference in the attenuator readings, $\Delta A_{L} \mathrm{~dB}$, is related to $\rho_{1}$ in logarithmic form,

$$
\Delta A_{L}=-20 \log \left(1+\rho_{1}\right) .
$$

The difference in the attenuator readings, $\Delta A \mathrm{~dB}$, in step (c) is related to $\rho_{0}$ in logarithmic form,

$$
\Delta A=20 \log \left(1+\rho_{0}\right) \text {. }
$$

From the values of $\rho_{1}$ and $\rho_{0}$ determined by Eqs. (A7) and (A8), $\gamma_{s}$ and $\beta$ are obtained through Eqs. (A3) and (A4).

In step (d) of the measuring procedure, the incident power on detector 2 is attenuated by $10 \log 2\left(1+\rho_{0}\right)^{2} /$ $\left[\left(1+\rho_{0}\right)^{2}+\left(1+\rho_{1}\right)^{2}\right] \mathrm{dB}$ instead of $3 \mathrm{~dB}$. Then, the loaded $Q$ is calculated by Eq. (8).

'C. G. Montgomery, Technique of Microwave Measurements (McGraw-Hill, New York, 1947), pp. 285-293, 309-319, 330-342, 396-407.

${ }^{2}$ E. L. Ginzton, Microwave Measurement (McGraw-Hill, New York, 1957), pp. 391-434.

${ }^{3} \mathrm{M}$. Sucher and J. Fox, Handbook of Microwave Measurement, 3rd ed. (Polytechnic Press, New York, 1963), pp. 417-494.

${ }^{4} \mathrm{~J}$. R. G. Twisleton, Proc. Inst. Electr. Eng. 103B, 339 (1956).

${ }_{5}^{5}$ A. Yariv and F. D. Clapp, Rev. Sci. Instrum. 30, 684 (1959).

${ }^{6} \mathrm{~J}$. R. Ashley and F. M. Palka, Microwave J. 14, 35 (1971). 